\title{
Making a bad relationship good
}

\author{
There is increasing evidence of direct and/or indirect interactions between bacteria and viruses. Two new studies \\ shed light on the mechanisms underlying these interactions with implications not only for our understanding of \\ microbial pathogenesis, but also for vaccine design.
}

\section{Karl J. Staples}

W ith the advent of modern molecular microbiology techniques, it is becoming increasingly clear that the lungs are not a sterile environment, even in the absence of lung disease ${ }^{1}$. There is evidence of co-existence, and thus co-evolution, of bacterial communities and the vertebrate immune response throughout history ${ }^{2}$, but recent data also suggest a similar interaction between these colonizing microorganisms and the evolution and pathogenesis of viruses $^{3}$. This seems especially true for the interaction between influenza virus and Streptococcus pneumoniae (Spn), as co-infection with these two pathogens results in worse disease symptoms and increased mortality than infection with either pathogen alone ${ }^{4}$. Two new studies ${ }^{5,6}$ in this issue of Nature Microbiology demonstrate direct interactions between Spn and influenza that have implications not just for our understanding of pathogenesis, but may also hold the key to the development of effective vaccines to prevent such infections.

Rowe et al. ${ }^{6}$ not only provide indirect evidence for a physical interaction between Spn and influenza by co-sedimentation, but also demonstrate direct evidence for binding of the virus to the bacteria by utilizing an influenza strain that expressed the mRuby 2 fluorescent protein ${ }^{6}$. To investigate what the impact of this direct binding of virus to Spn has on respiratory infection, these investigators demonstrated increased adherence of this viral-bacterial complex to in vitro-cultured human epithelial cell lines and in mice intranasally inoculated with Spn alone or Spn pre-incubated with influenza (Fig. 1, left panel). After just 24 hours, there was significantly more Spn recovered from the nasal passages and middle ears of mice inoculated with the pre-complexed Spninfluenza than when the two agents were administered without pre-incubation. The group then went on to demonstrate that the consequence of this increased binding of the complexed pathogens was a significant increase in mortality and the speed at which animals succumbed to infection.

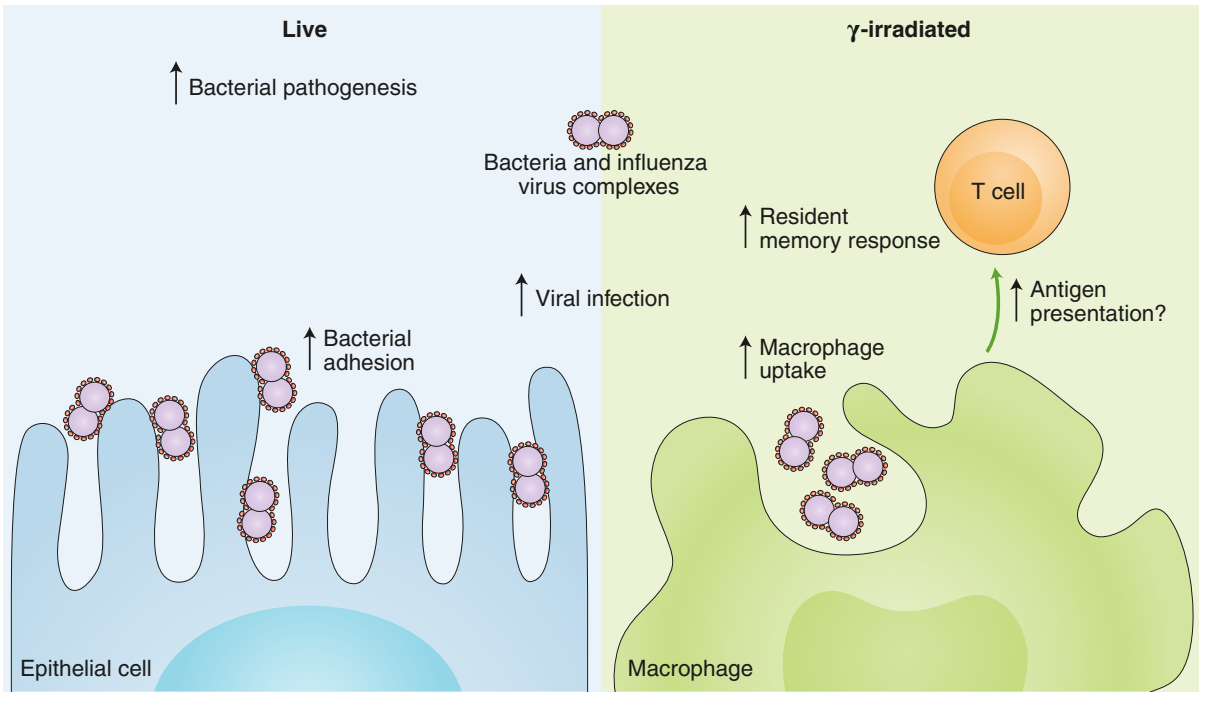

Fig. 1 | Interactions between influenza virus and Spn. Influenza virus directly binds to the surface of the bacterium, leading to increased adhesion of the bacteria to the respiratory epithelium, resulting in increased pathogenesis and mortality in a murine model. This viral binding to the bacterium is independent of bacterial viability, and use of a $\gamma$-irradiated Spn also resulted in increased viral infection of MDCK cells (left panel). When both Spn and the influenza virus were $\gamma$-irradiated, this direct binding between the two pathogens was still evident and led to increased uptake by macrophages. Mucosal vaccination of a murine model with this $\gamma$-irradiated pathogen complex enhanced lung resident memory T cell responses to influenza, possibly as a result of increased antigen presentation by macrophages (right panel).

Intriguingly, this increase in mortality was not associated with increased bacteraemia, suggesting that this disease enhancement may be a result of a cytokine storm that originates in the respiratory tract. But the question remains as to how this bacterialviral interaction benefits the virus.

David and colleagues in Australia have gone some way in providing an answer to this question. Not only do they present electron micrographs to further support the evidence of direct binding of influenza to a gamma-irradiated Spn ( $\gamma$-Spn) strain, they also demonstrate that this influenza- $\gamma$-Spn complex increases the ability of influenza to infect Madin-Darby canine kidney (MDCK) cells $^{5}$ (Fig. 1, left panel). While these investigators did not analyse if this increased viral infection was due to increased adherence of the bacterial-viral complex to the MDCK cells, they did demonstrate that the complex resulted in greater influenza uptake and internalization by THP-1 macrophages (Fig. 1, right panel). Thus, it appears that this interaction of influenza and Spn benefits infection of the host by both organisms, but could this interaction be exploited to enhance immunogenicity to these two pathogens? This Australian group of researchers has previously demonstrated that mucosal co-vaccination with a $\gamma$-irradiated influenza A/Puerto Rico/8/1934 (PR8) H1N1 strain ( $\gamma$-Flu) and $\gamma$-Spn resulted in significant protection against lethal co-infection and increased induction of Spn-specific responses ${ }^{7}$. The current paper concentrates on investigating the influenza-specific immunity that 
results from this $\boldsymbol{\gamma}$-Flu- $\boldsymbol{\gamma}$-Spn intranasal inoculation ${ }^{5}$.

Three weeks after vaccination with $\gamma$-Flu- $\gamma$-Spn, David et al. challenged mice with a lethal dose of the PR8 strain of influenza ${ }^{5}$. Unsurprisingly, mice vaccinated with $\gamma$-Flu alone or $\gamma$-Flu- $\gamma$-Spn were completely protected for up to three weeks post-infection, whereas mice that were mock-vaccinated or vaccinated with just $\gamma$-Spn alone succumbed to infection within one week of challenge. Mice vaccinated with $\gamma$-Flu alone or $\gamma$-Flu- $\gamma$-Spn were also protected from the effects of lethal challenge with the recent 2009 pandemic $\mathrm{H} 1 \mathrm{~N} 1$ strain. Importantly, only mice vaccinated with $\gamma$-Flu- $\gamma$-Spn were fully protected from the effects of challenge with the H3N2 heterosubtype, while there was $40 \%$ mortality in mice vaccinated with $\gamma$-Flu alone. Interestingly, David et al. demonstrated that this co-vaccination was not associated with a difference in either neutralizing antibody responses or circulating $\mathrm{T}$ cell responses. In contrast, $\gamma$-Flu- $\gamma$-Spn vaccination significantly enhanced resident memory $\mathrm{CD} 4^{+}$and $\mathrm{CD}^{+}$ $\mathrm{T}$ cell responses in the lungs, both of which are needed to confer protection to influenza infection $^{8,9}$ (Fig. 1, right panel).

Such interactions are not restricted to Spn, as Rowe et al. also provide evidence of direct interactions between influenza and other respiratory microorganisms, such as non-typeable Haemophilus influenzae (NTHi) and Moraxella catarrhalis ${ }^{6}$. These data may explain the observation that patients with chronic obstructive pulmonary disease who are colonized with NTHi are three times more likely to experience an exacerbation of their disease following viral infection than those who are not colonized ${ }^{10}$.

These studies by Rowe et al. and David et al. are not without their limitations. Not least that the majority of observations are derived from in vitro experiments or from lethal animal models rather than models that replicate human disease. In the paper by David et al., further work demonstrating the duration of protection will also be needed before these observations can be considered ready for translation to humans. Both papers also concentrate on the interaction between bacteria and influenza A strains, omitting mention of influenza B strains, even though B strains are an important circulating cause of influenza ${ }^{11}$. However, the results of the combined mucosal vaccine are tantalizing and could also be applied to the development of a vaccine to respiratory syncytial virus, which is already known to directly bind to $\mathrm{Spn}^{12}$. If the cross-protection to heterosubtypic strains can also be demonstrated for rhinovirus, then perhaps there may also be hope for a vaccine for the common cold.

Vaccine development is very much for the future, but for now the observations from Rowe et al. and David et al. should at least cause us to pause and consider how we will investigate respiratory infections going forward. While single organism infections may still be appropriate in many cases, given the pre-existence of the lung microbiota, co-infection rather than sequential infection models may be closer to reflecting the true situation of the lung.

\section{Karl J. Staples (D) 1,2}

${ }^{1}$ Clinical \& Experimental Sciences, University of Southampton Faculty of Medicine, Sir Henry Wellcome Laboratories, Southampton General Hospital, Southampton, UK. ${ }^{2}$ Wessex Investigational Sciences Hub, University of Southampton Faculty of Medicine, Southampton General Hospital, Southampton, UK.

e-mail:k.staples@southampton.ac.uk

Published online: 23 July 2019

https://doi.org/10.1038/s41564-019-0516-4

References

1. Hilty, M. et al. PloS ONE 5, e8578 (2010).

2. Pfefferle, P. I. \& Renz, H. F1000Prime Rep. 6, 11 (2014)

3. Karst, S. M. Nat. Rev. Microbiol. 14, 197-204 (2016).

4. McCullers, J. A. Nat. Rev. Microbiol. 12, 252-262 (2014).

5. David, S. C. et al. Nat Microbiol. https://doi.org/10.1038/s41564019-0443-4 (2019).

6. Rowe, H. M. et al. Nat. Microbiol. https://doi.org/10.1038/s41564019-0447-0 (2019).

7. Babb, R. et al. Clin. Sci. 131, 169-180 (2017).

8. Nakanishi, Y., Lu, B., Gerard, C. \& Iwasaki, A. Nature 462, 510-513 (2009)

9. Wilkinson, T. M. et al. Nat. Med. 18, 274-280 (2012).

10. Wilkinson, T. M. A. et al. Thorax 72, 919-927 (2017).

11. van de Sandt, C. E., Bodewes, R., Rimmelzwaan, G. F. \& de Vries, R. D. Future Microbiol. 10, 1447-1465 (2015).

12. Smith, C. M. et al. Am. J. Resp. Crit. Care 190, 196-207 (2014).

Competing interests

The author declares no competing interests. 